\title{
Téoros
}

Revue de recherche en tourisme

\section{L'histoire franco-belge du tourisme : une connaissance en voie de développement...}

\section{André Hut}

Volume 14, numéro 2, été 1995

Le tourisme : toute une histoire!

URI : https://id.erudit.org/iderudit/1075104ar

DOI : https://doi.org/10.7202/1075104ar

Aller au sommaire du numéro

Éditeur(s)

Université du Québec à Montréal

ISSN

0712-8657 (imprimé)

1923-2705 (numérique)

Découvrir la revue

Citer cet article

Hut, A. (1995). L'histoire franco-belge du tourisme : une connaissance en voie de développement... Téoros, 14(2), 48-50. https://doi.org/10.7202/1075104ar d'utilisation que vous pouvez consulter en ligne.

https://apropos.erudit.org/fr/usagers/politique-dutilisation/ 


\title{
L'histoire franco-belge du tourisme: une connaissance en voie de développement...
}

\author{
André Hut ${ }^{*}$
}

Les activitếs du signataire, depuis 35 ans, dans le tourisme, tant au niveau de l'animation sur le terrain qu'à celui de la recherche et de l'enseignement, l lui permettent de constater le sous-développement de l'histoire du tourisme dans son pays. L'inven= taire qui suit partage les fruits de l'investigation déployée en permanence ainsi qu'à Foccasion de la rédaction de mémoires universitaires de second et troisième cycle: il constitue un appel à prendre le relais et à creuser davantage le sillon...

\section{Du tourisme de station...}

Alors quele tourisme vient d'atteindre cent ans d'existence, Pierre Defert, géographe français, formule, en 1960 , les premiers repires historiques et une chronologie des dates importantes concernant l'évolution du tourisme en France et dans le monde ${ }^{(1)}$. Un collègue et compatriote, Marc Boyer, complétera et développera, la décennie suivante, cette chronologie et dressera un tableau synoptique comparant publications, organisations et associations, législations ayant trait au tourisme(2). De plus, il approfondira les analyses de son prédécesseur en explicitant la succession d'une classe sociale à l'autre: du tourisme aristocratque au tourisme social, en passant par le tourisme bourgeois, comme l'intitulera Arthur Haulot, commissaire gênéral au tourismeen Belgique, dans sa communication, au cours de la Journée scientifique organisée â l'occasion du $10^{*}$ anniverșaire de l'Institut international d'hôtellerie de Glion, en Suisse, en $1973^{\circ}$. Le second article présenté par le signataire $L e s$ vaances, retour anx sourcer, explicite ce processus (voir autre texte de André Hut dans ce numéro).

La même année, paraissait Le tourisme sur le littoral belge, aujourd'hui et demain, rédigé par Norbert Vanhove, du Buresud'étudeséconomiques de Flandres, dont le premier chapitre esquissait l'histoire du tourisme et ses conséquences sur la population du littoral ${ }^{(4)}$. Quinze ans plus tard, le Service culturel de la Caisse Géné-

Monsieur André Hut est, de la Belgique, le correspondant regulier de Tororos pour l'Europe rale d'Épargne et de Retraite (CGER) met sur pied une exposition, Histoire d'eatux. Stations thermales et balnéairer en Belgique du $X V{ }^{r}$ au $X X^{i}$ siècle, qui démontre, grâce à la recherche de l'historien Ronny Gobyn, comment il existe une filiation culturelle entre la station thermale ou la classe aristocratique se contente de transporter les comportements et loisirs de la cour et la station balnéaire qui reproduit les mếmes modèles et pratiques culturelles; de plus, un excellent collectif de rédacteurs poursuit et développe l'histoire des principales stations thermales et balnéaires du fittorals, déjà amorcée par le précédent auteur. La célébrité internationale et pluriséculaire des cures thermales de la villede Spa, située dans la partie wallonne, au sud-est du pays, est également évoquée par Étienne Helin dans le catalogue ${ }^{(9)}$.

Celui-ci fait écho à un ouvrage d'Armand Wallon ${ }^{69}$, paru en 1981, traitant de La vie quotidienne dans les villes d'eaux de France, de l'Antiquité au second Empire. Ce livre apporte la démonstration qui confirme la pertinence de l'analyse concernant le processus de reproduction culturelle mentionné ci-dessus. Coup sur coup, paraissaient à la même époque que le précédent, deux contributions, l'une, dans la même collection, $\mathrm{La}$ vie quotidienne sur les plages normandes, du second Empire aux années folles de Gabriel Desert, $1983^{\circ}$, l'autredans la concurrence d'une autre maison d'édition, intutulée Quand les grands duc valsaient à Nice, de Paul Auger ${ }^{\infty}$.

En parallèle, il faut brievement faire allusion à la montagne, au sport d'escalade et à la naissance du Club Alpin Français, en 1874, dont rend compte, par exemple, Les folles années de Chamonix de Gaby Curral-Couttet, dans la collection Si 1900 m'était conté (1984), la mểme année où la Suisse celébrait le centenaire des sports d'hiver, inaugurés déjà à Saint-Moritz, 25 ans avant la création du Davos English Ski Club, en $1902^{(9)}$.

Enfin, on aurait tort de sous-estimer l'énorme influence qu'ont eu sur le déploiement du tourisme, de grands événéments culturels comme les expositions universelles ou la résurgence des Jeux olympiques d'été et d'hiver: Quand Paris allait àl'Expo, 1855-1937 deJean-Jacques Bloch et Marianne Delort et plus particulièrement, L'Expo universelle de 1889 de Pascale Ory ou Jeux olympiques à Berlin, 1936 de Jean-Marie Brohm ${ }^{(09)}$.

\section{... à l'éclatement spatial}

En effet, si le tourisme de station, abordé ci-dessus, était tributaire d'un nouveau mode de transport, le chemin de fer, la démocratisation de l'automobile, après la Deuxième Guerre mondiale, grâce aux progrès techniques faworisés par celle-ci, va contribuer au tourisme dit de masse, notamment par la création de vastes et populeux campings sur tous les littoraux et fleuves d'Europe. Si ce sport et séjour de plein air a été pratiqué d'abord par une élite, depuis la fondaton du premier camping club de Londres, en 1875 , et celui de France, en 1910, il représentera, plus tard, le mode d'hébergementlemeilleur marché et le plus populaire. Conséquemment, la voiture entrainera un éclatement de l'espace touristique, puisqu'elle permettra d'atteindre n'importe quel lieu du territoire, er provoquera une dispersion incon trôlée des équipements. Le livre de Louis Montagne, Le camping ${ }^{(1)}$ en restitue l'histoire, l'évolution et la démocratisation progressive. Le même processus se déclenche lorsque les classes dominantes abandonneront progressivement celui-ci aux congés payés pour se toumer vers la seconde résidence et les vacances en milieu rural: chambres d'höte, gites, campingà la ferme. Henry de Farcy et Philippe de Gunzbourg ${ }^{(12)}$, dans Tourisme et milieu rural, y démontreront, dès 1967, les dimensions économiques, sociales, cul turelles et ludiques du logement chez l'agricul teur, des stations vertes de vacances sans oublier les offres qui proposent les parcs naturels régionaux.

La révolution culturelle de 1968 alimentera, notamment, le mythe d'un certain *retour à la natures non seulement à l'occasion des vacances, qu'abordent, par exemple, en 1977, Michel Marné et Jean Viard, dans La campagne inventée ${ }^{(11)}$, mais aussi, d'une manière ponctuelle, lors des 
fins de semaine, gräce aux Balades en Wallonie rurale proposées, en 1982, par Maurice Piraux, dans son guide C'est la ville qui nous envie ${ }^{(14)}$, ou encore l'engouement en faveur des randonnées, soit pédestres, en empruntant les anciennes lignes de chemin de fer, avec le guide de Gilbert Perrin, Chemins de traverses, en $1993^{(15)}$, soit, à vélo, le long des canaux que recommandent déja Gêrard de Selys et Anne Maeschalk, en 1989, dans Cyclotourisme en Belgique. $1000 \mathrm{~km}$ de chemins royaux $x^{(10)}$. A ce propos, signalons les diver ses possibilités de séjours-croisières que commercialise, avec succès, depuis une dizaine d'années, le tourisme fluvial.

Enfin, à l'automobile s'ajoute, après 1950, un nouveau transport, à présent collectif, l'avion commercial et le développement des voyages organisés, en groupe. Ceux-ci se concentrent dans les stations touristiques classiques comme aussi se dispersent le long des littoraux et/ou dans la montagne.

\section{Métiers et politiques}

Si les ouvrages précédents jalonnent une approche de l'évolution des hébergements et des transports touristiques ainsi que des choix et des pratiques, notamment culturelles, des vacanciers, il n'existe pratiquement rien, en circulation, sur l'histoire des syndicats d'initiatives, dont certains ont été fondés il y a cent ans déjà, des Fédérations provinciales du tourisme, pas plus que celle de la politique des ministres successifs qui ont eu ce secteur d'activités en responsa bilité, du Commissariat général qui avait mission d'exécuter celle-ci ou de l'Office chargé de sa promotion.

Quant aux métiers touristiques, quelques autobiographies de travailleurs de l'aviation - pilote de ligne, hôtesse de l'air - ou de paquebots de croisières ${ }^{(17)}$ ouvrent un champ d'investigation encore inexplorế. Marcel Gautier, digne représentant de sà profession, le constate et le regrette aussi dans son livre-témoignage: L'industrie hôtelière. Le défi a été relevé par Joseph Seydoux, quand - accompagné d'un calendrier historico-politique du tourisme, qui bonifie les précédents et une vaste *bibliographie historiquess sur le sujet - il développe une approche historique ample et fouillée De l'hospitalité à l'accueil, à laquelle Serge Perrot, ancien directeur et inspecteur de l'enseignement hôtelier, apporte d'originaux complément ${ }^{(11)}$. Quant à l'étude sociologique étayée de précieuses références bibliographiques, réalisée par un maiture d'hôtel, J.O. Retel, Les gens de l'hôtellerie, clle révèle les conditions de travail, les valeurs humaines et les qualités professionnelles vécues par ces derniers et par trop ignorées en dehors du milieu ${ }^{(19)}$.

En outre, dans Agences et associations de voyages, Robert Lanquar ${ }^{D 0}$ éveille l'appétit des chercheurs, en présentant l'inventeur de ce métier, le célèbre Thomas Cook, les initiatives des autocaristes et, enfin, le fondateur de la compagnie des wagons-lits, un belge, fils de banquier, Goorges Nagelmackers: Jean des Cars raconte les réussites et les avatars de ce. capitaine qui tient la barre de certe exceptionnelle entreprise de services ${ }^{(2)}$. Si un chapitre de ce dernier auteur nous fait mieux connaitre les fonctions et les responsabilités del'employé, leconducteur, chargé del'accueil et de la gestion d'un wagon-lit, le spécialiste Henri Vincenot, dépeint, de l'intérieur, $\mathrm{La}$ vie quotidienne dans les chemins de fer au XIX siècle (22), de l'ensemble des métiers regroupés dans la corporation des cheminots; de plus, les principales dates de l'histoire des chemins de fer au XIX' siècle et la bibliographie très circonstanciée, situent ces professions dans l'évolution de ce moyen de transport particulièrement marié au tourisme.

\section{Formations et concertations}

Il est assez significatif que des compléments de licence, de dewième cycle, abordant le tourisme, n"aient été offerts que depuis cinq ans seulement dans des universités des deux régimes linguistiques du pays. Le milieu scientifique n'a pas encore vraiment pris le tourisme au sérieux; de plus, celui-ci est une réalité complexe, à la fois sociale, économique, juridique, culturelle, historique, environnementale, politique, éthique et spirituelle, appelant, pour l'appréhender dans la totalité de ses composantes, des approches complémentaires et pluridisciplinaires, ce qui n'est pas habituel dans une institution où les facultés sont aussi cloisonnées!

D'ailleurs, les professeurs diplồmés qui enseignent les matières liées au tourisme - français, langues étrangères, géographie, économie, législation, philosophie ou morale - dans les niveaux du secondaire technique (15-18 ans) ou du graduat (18-21 ans)spécialisésen ce domaine, n'ont pasreçu de formation spécifique; ils doivent l'acquérir par eux-mémes: il n'existe pas de formation continue appropriée et les bibliothèques des écoles n'ont pas toujours la quantité et la qualité scient tíques voulues. Il y a certes déjà eu des thèsesuniversitaires qui ont abordé des sujets touristiques, no- tamment en histoire, mais, jusquu'à présent, celles-ci ne jouissaient d'aucune publicité systématiséeetrestaient confidentiellement classées et dispersées géographiquement au gré de chaque institution d'enseignement. Depuis peu, cela va changer: à la suite de l'initiatve prise par le Commissariat au tourisme de Flandres, le nouveau et récent Bureau bruxellois de promotion du tourisme (BBPT) va créer un Centre de documentation et d'information quidevra, sclon le Livre blan e'13) édité à la fin des Assises du tourisme bruxellois: wil devra réunir toutes les statistiques et publications relatives au tourisme, $y$ compris les mémoires et thèses des étudiants qui $y$ sont consacrés $*$ (p. 24).

Enfin, il manque un Centre national de recherche en histoire du tourisme dont les multiples tâches et fonctions pourraient permettre, stimuler et coordonner les travaux, l'information et les concertations nécessaires au service des étudiants des facultés, des chercheurs et du secteur touristique $f$

\section{NOTES}

(1) DEFEAT, Pierre, Pour une politique du touriame en France, Paris, Editions Oumieres, 1960.

(2) BOYER, Marc, Le tourisme, Paris, Seuil, collection Peuple et cullure, 1972

(3) HAULOT, Arthur, Du fourisme aristocra tique au fourisme cocia! in Revue de l'AIEST (sur leméme themel. Berne, Suisse, Editions Gunten, vol. 14, 1973.

(4) VANHOVE, Norbert, L histoire du tounsme of ses consequences sur la population du littoral, tiré de consequences sur a population du ittorar, tire de Le tourisme wur le littoral belge, aujourd hui et
demain. Bruxelles. Editions du eredit communal. demain, Brukelles, Editions du credit communal,
collection Sciences sociales. Pro civitatae, no 3. collection
1974 .

(5) Histoire d'eaus. Stations thermales et baineaires en Belgique du $X^{\prime} V^{*}$ au $X X^{*}$ siecle; titre d'une exposition organisee a Bruxelles en 1967 par b CGEF Le calalogue de cette emposition conten des textes de difterents auteurs sur "histoire des principales stations du littoral et celui d'Étienne principales stations du littoral et celit

(6) WALLON. Armand, La vie quotidienne dans les villes d'eaux de France de I'Antiquite au vecond Empir . Paris, Hachette, 1981.

(7) DESERT, Gabriel, La vis quotidienne sur las plages normandes du second Empire aux années follos. Paris, Hachette, 1963.

(18) AUGIER, Paul, Ouand les grands ducs valsaient a Nice, Paris, Fayard, 1983

(9) CURAAL-COUTTET, Gaby, Les follew annees de Chamonix. Paris, france-Empire, collection - Si Chamonix. Paris, france-Em
1900 m etat conten, 1984 .

(10) BLOCH, Jean-Jacques et Marianne DELORT, Quand Paris allait a IExpo, 1855-1937. Paris. Marabout, Fayard, 1960; Ory. Pascale, L'Exposition univer selle de 1889 . Bruxelles, Editions Complexe, no 210, 1989, Brohm, Jean Marie, Le: jeux olymplques a Ber lin, 1936, Bruxelles, Edi hons Complexe, no 25. 1983.

(11) MONTAGNE, Louis, Le camping, Paris, PUF, collection Que sais-e? no $1594,1975$.

(12) DE FAACY, Henry et Philppe DE Gunzbourg. Tourisme ot milieu rural, Pans, Flammarion, collection La Terre, 1967.

(13) MAANÉ, Mothel et Jean Vurd, La campagne in. ventbe, France, collection Espace-Temps, Editions Actes-Sud, 1977

(14) PIRAUX. Maurice, C'est la ville qui nous envie, Arlon, Belgique, Fondation Furale de Wallonie, 1982

(15) Pernin, Gibert, Chemins de traver ses, Brukelles, ATBF, Fondation Roi Beaudouin. 1993. 
(16) DE SELYS, Gerard et Anne Maeschalk, Cyclotourisme en Belgique. $1000 \mathrm{~km}$ de chemins royaux, cosditions buculot et Touring Club de Beigique, 1989

[17] PETIT, Edmond, La vie quotidienne dan l'avia. tion en France as dibut du $X X *$ aibele, 1900. 1935, Paris, Hachette, 1977; GREARD, Andre, Le métier de pilote de ligne, Paris, France-Empire. 1979: MAHUZIER, Alain-Pierre, commissaire de bofd. Tras chere France, Paris, Stock 1978.

(18) GAUTHIEA, Marcel, L'industrie hóteliare, Paris PUF, collection Que saiste?, no 1022, 1970 U F , praccueit Denges (Suisse), collection Delta et Spes, 1980. Perrot, Serge, La mótier i du tourisme, Paris. Editions Lanore, 1977.

(19) RETEL, J.O., Les gent de rhótellerie, Paris, Editions ouvneres, collection L'évolution de la vie sociale, 1965.

(20) LANQUAR Rober, Agences et associations de voyages. Paris. PUF, collection Que saisje?, no 1787,1979 .

121) DESCARS, Jean Sleoping Story, Paris, Editions $J$ at Lu, no 832,1976

[22] Vincenot, Henti, La vie quotidienne dane les chemins de fer au XIX* siecle, Paris, Hachette. 1975.

(23) PILLEN, Peter, ct, Office de promotion du tourisme, Bruxelles. 1995 .

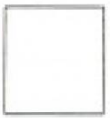

Del'histoire individuelledesvacances, il faut passer à l'histoire collective de celle-ci. En effet, à force de les vivre chaque année comme un fait acquis, on en arriverait à oublier qu'elles n'ont pas toujours existé et que les classes sociales y ont accédé les unes après les autres.

La classe aristocratique d'abord; les familles royales et leurs suites - noblesse et courtisans - de 1850 à 1914. Lorsque celle-ci est ruinée par la guerre et exclue du pouvoir, quasi partout en Europe, par les bouleversements politiques, une autre classe prend le relais et lui succède: la bourgeoisie industrielle. Enfin, d'une manière généralisée, l'action collective du mouvement ouvrier conquiert, dans les divers pays d'Europe, le droit au tourisme social. Ainsi que le définit Arthur Haulot :

Par tourisme social, nous entendons le phénomène qui a marquél'accè à lactivité touristique - ence compris le voyage et le sejour - de couches sociales qui jusque-là en avaient été tenues économiquement à l'écart. Les raisons de cet éloignement sont à trouver en effet, non dans une disposition d'esprit particulière, un choix dilibéré, un refus motivé ou une quelconque *incapacité culturellew mais bien dans les conditions prévalant à tune époque donnée dans l'organisation économique et sociale de la production. Cette organisation se traduisait, jusqu'en 1936, et pour la plus grande partie de la population active de nos payseuropéens, par l'absence de deux ingrédients deja énoncés: le temps

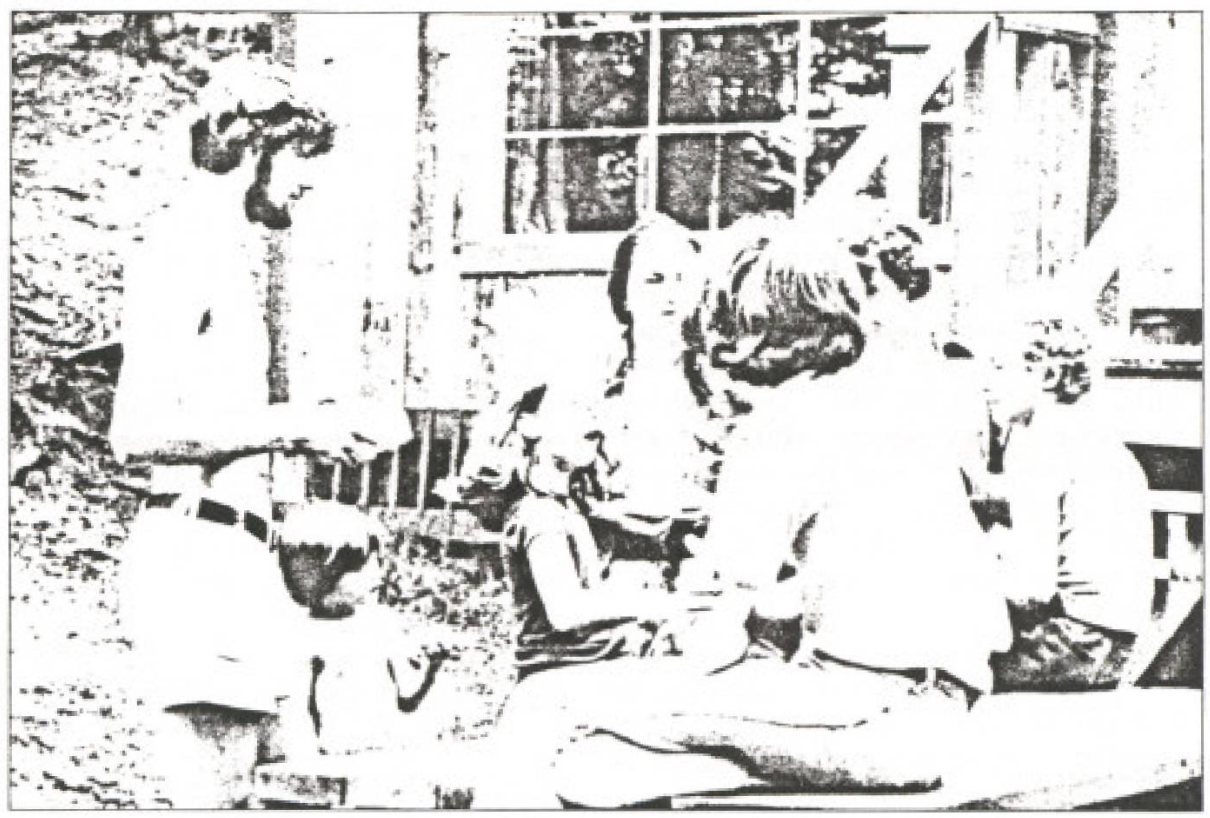

\section{Vacances... retour aux sources!}

\section{De la conquête... à la régression}

André Hut

que n'octroyait pas le rytbme de travail ni bebdomadaire ni annuel, et largent, que n'assurait pas le niveau salarial moyen, ni le revenu familial. La législation a reconnu et proclamé le droit au temps libre des vacances annuelles et ce geste politique fut la traduction d'une evolution sociale et pbilosopbigue extraordinaire autant qu'irréversible. Pour la première fois dans l'ere industrielle, L qualité d'bomme venait se superposer à celle d'outil bumain, la dignité d'être était reconnue indépendamment de la capacité de produire.

\section{Face à des loisirs commercialisés}

Malheureusement, les chances de libération et d'épanouissement culturels que peuvent offrir les vacances aux classes populaires sont sérieusement compromises par l'industrialisation intensive des loisirs et du tourisme dans les stations balnéaires rassemblant de massives concentrations de vacanciers surles littoraux européens. Dans un quasi-désert culturel, lesvacanciers sont confrontés aux loisirs commercialisés: *dancings $*$, jeux publicitaires, fêtes foraines bruyantes et permanentes, piscines tropicales.
Les parcs d'attractions représentent l'alternative rentable pour les managers touristiques. En conséquence, les projets se multplient: Walibi, Schtroumpf, Astérix et autres Disneyland en gestation. Qu'offrent-ils sinon des loisirs passifs et mécaniques, prostituant l'imaginaire des héros de bandes dessinées dans le voyeurisme d'un spectacle de la consommation et de la pseudo-fête artificielle?

\section{Le tourisme associatif}

Ces initiatives se caractérisent par leur statut juridique d'association sans but lucratif à l'opposé des entreprises commerciales, leurs contributions à l'épanouissement personnel des participants, enfin leur démarche pédagogique d'implication de ces derniers au déroulement de l'activité et même au projet.

\section{Le tourisme social}

Parmi celles-ci, le tourisme appelé *social $\gg$ s'en distingue par ses origines historiques, sa volonté de développement et d'épanouissement, non seulement individuel mais aussi collectif, des personnes appartenant aux classes populaires. Enfin à cause d'une réglementation légale qui lui est propre (relative à l'allocation de subventions en vue de promouvoir les vacances ouvrières et le tourisme populaire). 